\title{
Second International Conference on Advances in Pulmonary Rehabilitation and Management of Chronic Respiratory Failure, Venice, 4-7 November 1992
}

\author{
M D L Morgan
}

In Britain pulmonary rehabilitation has never achieved the status or respect which is customary in North America or continental Europe." It is more likely to be available in countries with consumer driven health care services but the failure of development may also be because it is a poorly defined activity whose outcome is difficult to measure. During the last year there have been two major contributions to the re-establishment of the concept of pulmonary rehabilitation in the mainstream of respiratory medicine. The first was the publication of a supplement to the European Respiratory fournal which described the depth and variety of those activities which constitute pulmonary rehabilitation. $^{1}$ The other was the second International Conference on Advances in Pulmonary Rehabilitation and Management of Chronic Respiratory Failure, which was held in Venice in November 1992. Delegates from 25 countries heard how advances made in the several years since the first conference have made rehabilitation a more respectable practice.

Rehabilitation is defined as the maximisation of a patient's physical, mental, social, and vocational potential. In other words the aim is to achieve adaptation of a patient's lifestyle, over and above conventional pharmacological treatment, which improves the quality of life. This will involve the coordinated application of several interventions by the appropriate health service professionals, the degree of which will depend on the patient's need. Rehabilitation is thus multidisciplinary and includes components such as oxygen therapy, domiciliary ventilation, physical exercise, nutrition, psychological support, and antismoking advice. The conference covered sessions on all these elements as well as presenting comparisons of practice in different countries.

There has always been a major credibility problem with pulmonary rehabilitation as patients have claimed to feel better as a result of the interventions but this has not been

\footnotetext{
Department of Respiratory Medicine, Glenfield Hospital, Leicester LE3 90P M D L Morgan
}

In an attempt to encourage a higher profile for rehabilitation in chronic obstructive lung disease there will be a review series in Thorax in 1993.-Editor reflected by improvements in static lung function or maximal exercise performance. Although the conference covered diverse subjects, many of which are still on the fringe, it may be remembered for focusing on the correct methods of identifying the benefits of rehabilitation programmes.

There was, understandably, an emphasis on chronic obstructive lung disease with plenary reviews of the natural history and pathophysiology of the condition. In spite of its much studied status there are many unanswered questions concerning the development of respiratory failure in chronic obstructive pulmonary disease. One of the more interesting is the relationship between respiratory muscle function and respiratory control as described by Peter Macklem who suggested that fatigue avoidance may be a major objective of respiratory control. This would certainly explain why fatigue has never been satisfactorily demonstrated in the clinical situation. Support for patients in respiratory failure has traditionally included oxygen therapy, which has been given uncritically since the MRC and NOTT trials. It was suggested, however, that domiciliary oxygen may not necessarily improve the quality of life in patients who are severely enough affected to satisfy the criteria for long term oxygen therapy, and consideration should be given to supplying oxygen to patients while they are still mobile and can benefit from it. Examination of the various national facilities for the provision of portable oxygen makes an embarrassing comparison with the UK's woeful record. An alternative treatment for respiratory failure in the form of nasal intermittent positive pressure ventilation is beginning to emerge but debates about its effectiveness without additional oxygen will have to wait for the outcome of the European multicentre trial being coordinated by Dr Muir from Rouen. There were other sessions on physical exercise and rehabilitation, with papers confirming that patients with respiratory disease often lack physical fitness, which can be improved even by home based training. We heard again that inadequate nutrition is associated with poor prognosis in lung disease but the relationship is not simple and correction may be difficult. 
The most important component of the conference was the inclusion of sessions on the quality of life. It is clear that the previously vague benefits of rehabilitation can now be quantified with a number of general utility and disease specific instruments. Internationally eminent speakers, including Gordon Guyatt and Paul Jones, emphasised that we now have the ability to assess the clinical and economic consequences of treatment. Historically pulmonary rehabilitation has fallen from grace because of an inability to measure its effects, but now its benefits can be subjected to trial and sensitive scrutiny. ${ }^{2}$ It may require very little physiological improvement to produce an appreciable increase in the quality of life. This conference and hopefully its successors will give confidence to clinicians who believe that they have more to offer than just the prescription of drugs.

1 Donner CF, Howard P, eds. Pulmonary rehabilitation in chronic obstructive pulmonary disease (COPD) with recommendations for its use. Eur Respir f 1991;1: review No 6.

2 Neiderman MS, Clemente PH, Fein AM, Feinsilver SH, Robinson DA, Ilowite JS, et al. Benefits of a multidisciplinary pulmonary rehabilitation program. Chest 1991;99:798-804.

\section{"Doping" with $\beta_{2}$ agonists in the 1960s?}

Olympic skiers were reported in 1992 to inhale $\beta_{2}$ agonists to help them win medals in the Olympic winter games in Albertville. Exercise and cold air induced airways constriction have been well known phenomena for many years. In the early 1960s I worked for a couple of years at the Laboratory for Respiratory Physiology at the University Lung Clinic, Renström's Hospital in Gothenburg, Sweden. After contact with Professor Tiffeneau around 1960 I started to test bronchial reactivity by acetylcholine inhalation provocation tests. Gunnar Grimby, well known for his work on rehabilitation of respiratory patients, at that time also treated some top athletes for various ailments, among them some skiers with apparent exercise induced bronchoconstriction. Some of these athletes were referred to me for testing and possible treatment. One of them held a number of Swedish championships in skiing and had won the original long distance ski race (Vasaloppet, over $90 \mathrm{~km}$ ) several times. He had obvious breathing difficulties, however, in cold weather, especially at short distances $(10-15 \mathrm{~km})$ which needed fast immediate speed and large minute ventilation. At that time we only had non-selective $\beta$ agonists, isoproterenol, and the slightly more selective orciprenaline for treatment of acute bronchoconstriction. Preventive inhalation of these dose aerosols before skiing had obvious benefits. Several top skiers have since used inhaled $\beta_{2}$ agonists to improve or keep up their performance. Exercise induced bronchoconstriction is thought to be one expression for bronchial hyperresponsiveness leading to bronchoconstriction. Recent studies suggest that bronchial hyperresponsiveness is very common among top athletes, especially skiers.

Recently a peroral $\beta_{2}$ agonist, clenbuterol, has been used as a protein anabolic and thermogenic agent in athletes. It seems unlikely that inhalation of $\beta_{2}$ agonists in the doses used for prevention of exercise induced bronchoconstriction have a similar effect. The benefit is more likely to result from inhibition of exercise and cold air induced bronchoconstriction preventing an increase in the work of breathing. Another type of prevention against cold and exercise induced bronchoconstriction appeared on the Swedish market in the 1980 s, namely air conditioning devices which warm and moisturise the inhaled air. They were extensions of the heat and moisture exchanger reported by Toremalm in 1960 for use after tracheotomy. Swedish pharmacies now offer such a device which is much used by athletes who need hard outdoor training all the year round despite cold weather. One such device is called "LungPlus" and represents an excellent non-pharmacological method of preventing exercise and cold induced airways constriction in our asthmatic patients who also benefit from the larger series manufactured for the larger number of training athletes.

BG SIMONSSON 